\title{
The relationship between hyperuricemia and contrast-induced acute kidney injury undergoing primary percutaneous coronary intervention: secondary analysis protocol for the ATTEMPT RESCIND-1 study
}

Wei Guo ${ }^{1,2+}$, Feier Song ${ }^{1 \dagger}$, Shiqun Chen ${ }^{1 \dagger}$, Li Zhang ${ }^{2}$, Guoli Sun ${ }^{1}$, Jin Liu ${ }^{1}$, Jiyan Chen ${ }^{1}$, Yong Liu ${ }^{1 *}$, Ning $\operatorname{Tan}^{1 *}$ and the RESCIND group

\begin{abstract}
Background: Contrast-induced acute kidney injury (Cl-AKI) contributes toward unfavorable clinical outcomes after primary percutaneous coronary intervention (pPCl). We will assess whether hyperuricemia is an independent predictor of $\mathrm{Cl}-\mathrm{AKI}$ and outcomes in patients undergoing $\mathrm{PPCl}$.

Methods/design: Our study is a secondary analysis for the database from ATTEMPT study, enrolling 560 STsegment elevation myocardial infarction (STEMI) patients undergoing $\mathrm{pPCl}$. Patients will be divided into 2 groups according to the admission serum uric acid (SUA) level. Hyperuricemia will be defined as a SUA level $>7 \mathrm{mg} / \mathrm{dL}$ $(417 \mathrm{mmol} / \mathrm{L})$ in males and $>6 \mathrm{mg} / \mathrm{dL}(357 \mathrm{mmol} / \mathrm{L})$ in females. The primary endpoint was $\mathrm{Cl}-\mathrm{AKI}$, defined as $>25 \%$ or $0.5 \mathrm{mg} / \mathrm{dL}$ increase in serum creatinine from baseline during the first $48-72 \mathrm{~h}$ post-procedurally. Multivariate analyses for $\mathrm{Cl}-\mathrm{AKI}$ and long-term mortality will be performed using the logistic regression and Cox regression analyses, respectively.

Discussion: This study will determine the predictive value of hyperuricemia for the development of $\mathrm{Cl}-\mathrm{AKI}$ and outcomes in patients with STEMI undergoing PPCl. We predict that hyperuricemia will be associated with a risk of $\mathrm{Cl}$-AKI in patients with $\mathrm{pPCl}$. Furthermore, after adjusting for other variables, long-term mortality after $\mathrm{pPCl}$ may be higher in those with hyperuricemia than in those with normouricemia. Results of this study may provide scientific evidence for the effect of hyperuricemia on $\mathrm{Cl}$-AKI and long-term outcomes, thereby offering the potential possibility of lowering SUA on the development of $\mathrm{Cl}$-AKI and outcomes.
\end{abstract}

Trial registration: ClinicalTrials.gov NCT02067195, Registered on 20 February 2014.

Keywords: Hyperuricemia, Contrast-induced acute kidney, ST-segment elevation myocardial infarction, Primary percutaneous coronary intervention

\footnotetext{
*Correspondence: liuyong2099@126.com; gdgtanning@163.com

†Wei Guo, Feier Song and Shiqun Chen contributed equally to this work.

'Department of Cardiology, Guangdong Provincial Key Laboratory of

Coronary Heart Disease Prevention, Guangdong Cardiovascular Institute,

Guangdong Provincial People's Hospital, Guangdong Academy of Medical

Sciences, Guangzhou 510080, China

Full list of author information is available at the end of the article
}

(c) The Author(s). 2020 Open Access This article is licensed under a Creative Commons Attribution 4.0 International License, which permits use, sharing, adaptation, distribution and reproduction in any medium or format, as long as you give appropriate credit to the original author(s) and the source, provide a link to the Creative Commons licence, and indicate if changes were made. The images or other third party material in this article are included in the article's Creative Commons licence, unless indicated otherwise in a credit line to the material. If material is not included in the article's Creative Commons licence and your intended use is not permitted by statutory regulation or exceeds the permitted use, you will need to obtain permission directly from the copyright holder. To view a copy of this licence, visit http://creativecommons.org/licenses/by/4.0/. The Creative Commons Public Domain Dedication waiver (http://creativecommons.org/publicdomain/zero/1.0/) applies to the data made available in this article, unless otherwise stated in a credit line to the data. 


\section{Background}

ST-elevation myocardial infarction (STEMI) patients undergoing primary percutaneous coronary intervention (pPCI) are commonly complicated by contrast-induced acute kidney injury (CI-AKI) [1]. CI-AKI is associated with higher hospitalization rates, long-term morbidity, and mortality [2]. Therefore, patients recognized with high-risk factors of CI-AKI should be carefully monitored and treated with appropriate prophylactic strategies. Based on previous study, chronic kidney disease, diabetes, hypotension, contrast volume, congestive heart failure, advanced age, and anemia have been identified as risk factors of CI-AKI [3].

Uric acid is the final product of purine metabolism, which is metabolized by xanthine oxidase [4]. Substantial evidence suggests that hyperuricemia is an independent risk factor for hypertension, metabolic syndrome, chronic kidney disease, and cardiovascular diseases [5]. In previous studies, we reported that hyperuricemia was an independent risk factor for CI-AKI after PCI $[6,7]$. However, the conclusion remains controversial [8-15]. In addition, only a limited number of studies reported that hyperuricemia was an independent predictor of CI-AKI in STEMI patients undergoing pPCI. Moreover, it was uncertain about the role of uric acid in the long-term outcome [16-18].

We aimed to investigate the association of hyperuricemia with CI-AKI in patients with high-risk STEMI undergoing pPCI and to determine the predicting role in prognosis.

\section{Methods/design}

\section{Study design and population}

This is a secondary analysis of aggressive hydration in patients with STEMI undergoing $\mathrm{pPCI}$ to prevent contrast-induced nephropathy, the first study for reduction of contrast-induced nephropathy following cardiac catheterization (ATTEMPT RESCIND-1 study). Five hundred sixty patients aged 18 years or older, treated with $\mathrm{pPCI}$, and provided written informed consent were included from 15 medical research centers in China. Inclusion and exclusion criteria were described elsewhere [19]. We also excluded the patients without the admission serum uric acid (SUA) level.

\section{Study protocol}

Baseline data will include demographics, diagnosis, medical history, laboratory parameters, medications, and physical examination. Patients will be divided into 2 groups according to the admission serum uric acid (SUA) level. Hyperuricemia will be defined as a SUA level $>7 \mathrm{mg} / \mathrm{dL}(417 \mathrm{mmol} / \mathrm{L})$ in males and $>6 \mathrm{mg} / \mathrm{dL}$ (357 mmol/L) in females. The comorbidities will include hypertension, diabetes mellitus, hyperlipidemia, heart failure, chronic kidney disease, stroke, peripheral arterial disease, and chronic obstructive pulmonary disease. The concomitant therapies were decided by the doctors and were relied on the routine standards of care recommended by the current guidelines. The primary outcome, CI-AKI, was defined as a $25 \%$ or $0.5 \mathrm{mg} / \mathrm{dL}$ increase in serum creatinine from baseline at $48-72 \mathrm{~h}$ after pPCI [19]. The secondary endpoints were different definitions of CI-AKI, persistent renal impairment, major adverse clinical events during hospitalization, total hospitalization costs, and length of hospital stay [19].

Follow-up adverse events will be recorded by trained investigators via office visits or telephone interviews at 3 , $6,12,18$, and 24 months after the pPCI. Long-term outcomes will be major adverse cardiovascular events (MACEs), including mortality, stent restenosis, non-fatal myocardial infarction, and target vessel revascularization.

All data will be collected with standardized electronic case report forms. At the time of enrollment, the data management team of Guangdong Provincial People's Hospital will conduct consistency checks and issues data clarification forms to deal with discrepant data. All data will be collected after the approval by the ethics committee of all participating centers. An independent data monitoring committee will review the ongoing safety events of every participant.

How data are stored and managed had explained in the "data and safety monitoring board" of our previous study [19]. The details are as follows. A committee of clinicians and a biostatistician, chaired by Chun Wang, $\mathrm{MD}$, and Chun-quan $\mathrm{Ou}, \mathrm{MS}$, who are blinded to the treatment allocation, will periodically review and evaluate the accumulated study data for participant safety, study conduct, and progress, and, when appropriate, efficacy and will make recommendations to the principal investigators concerning the continuation, modification of enrollment, or termination of the trial. Details of any protocol violations (e.g., descriptions, reasons, and resolution) will be reported by the investigators and clinical research associates to the primary investigators, the Central Event Adjudication committee, and Data and Safety Monitoring Board, if necessary. In addition, training will be provided to the researchers, and the study procedure will be supervised, with particular attention to the reasons for bias and data verification to avoid recurrence of protocol violations. Finally, intention-to-treat analysis will be used for the patients with protocol violations instead of the per-protocol analysis [19].

The literature review of hyperuricemia and CI-AKI has reported to the RESCIND group's academic committee. After that, a statistical scheme will be written and submitted to statisticians and data administrators to obtain data.

\section{Statistical analysis}

Baseline patient characteristics and information will be presented as the mean \pm standard deviation or median 
plus interquartile ranges of descriptive statistics. They will be compared by $t$ test or Wilcoxon rank sum test according to distribution. The categorical data about baseline characteristics and information will be expressed as a percentage were analyzed using the Pearson chisquare test or Fisher's exact test. The association between hyperuricemia and CI-AKI will be performed using multivariate logistic regression analysis. Multivariable logistic regression models have been developed to adjust for clinical characteristics (e.g., age, sex, estimated glomerular filtration rate, left ventricular ejection fraction, chronic heart failure, anemia, diabetes mellitus), and the Mehran risk score will be calculated [3]. All variables that are univariately associated with this outcome measure will be entered as possible predictors in a multivariable logistic regression analysis. The association between hyperuricemia with long-term mortality will be investigated by Cox regression model adjusting for other risk factors (e.g., age, CI-AKI, heart function, renal function, and medication). KaplanMeier curve and log-rank test will be performed on the survival time of the hyperuricemia group and the normouricemia group (the cumulative rate of all-cause mortality and MACEs between these two groups). These data will be analyzed on the basis of valid cases. A two-tailed $p$ value $<0.05$ will be considered statistically significant. The power calculation was based on our previous finding [20], and a CI-AKI incidence of $23 \%$ was estimated for the hyperuricemia group, while $11.5 \%$ was assumed for the normouricemia group (50\% relative reduction). Using a twosided chi-square test, a significance level of 0.05 , and a sample size of 280 each group, the power of the tests is over $90 \%$.

All statistical analyses will be performed using SAS version 9.4 or later (SAS Institute, Cary, NC, USA) and $\mathrm{R}$ software (version 3.1.2; R Foundation for Statistical Computing, Vienna, Austria).

\section{Discussion}

CI-AKI is closely associated with prolonged hospital stay, long-term morbidity, and mortality in patient undergoing PCI. The incidence of CI-AKI is about $2 \%$ in the general population and over $50 \%$ in high-risk population [3]. Previous study supported the relationship between CI-AKI and higher incidence of adverse short- and long-term cardiovascular outcomes, including mortality [21]. Patients with STEMI are likely to present with hypotension, or even cardiogenic shock, higher volume of contrast media, and impossibility of renal prophylactic therapy, which are associated with an increased risk of CIAKI [22].

Advanced age, diabetes, dehydration, hypotension, sepsis, cardiovascular disease, underlying acute kidney injury, chronic kidney disease, and concomitant use of nephrotoxic drugs were identified as well-known risk factors for CI-AKI $[3,23]$. In previous studies $[6,7]$, we reported that hyperuricemia was an independent risk factor for CI-AKI in PCI patients, which was consistent with other studies [8-13]. However, other studies did not show the same conclusion [14, 15]. Several studies explored the effect of serum uric acid (SUA) on CI-AKI among high-risk patients such as STEMI undergoing primary PCI. Elbasan et al. showed that SUA was associated with CI-AKI in STEMI patients undergoing pPCI (mean SUA $=6.2 \pm 0.9 \mathrm{mg} / \mathrm{dL}, 95 \% \mathrm{CI}, 1.877-3.236 ; p=$ 0.002) [16]. In another study, Mendi et al. demonstrated that SUA $\geq 5.4 \mathrm{mg} / \mathrm{dL}$ was an independent risk factor for CI-AKI (OR 1.26, 95\% CI, 1.10-1.42; $p<0.001$ ) [17]. Saritemur et al. showed that elevated uric acid was lined with CI-AKI in multivariate analysis after adjusting for potential confounding factors (OR 1.01, 95\% CI, 1.00$1.01 ; p=0.01$ ) [18]. However, even though they reported that high SUA was an independent predictor of CI-AKI in STEMI patients, there was no uniform standard for the definition of hyperuricemia. In our study, we will employ the definition consistent with our previous study.

In addition, these studies did not compare the prognosis between CI-AKI group and non-CI-AKI group in STEMI patients undergoing PCI. Some observational studies proved the relationship between hyperuricemia and clinical outcomes in the presence of gout, but not for asymptomatic hyperuricemia [24, 25]. Nevertheless, a recent study by Pagidipati et al., including the PLATO and TRACER study population, demonstrated a significant association between uric acid (UA) and short-term adverse outcomes, independent of the presence of gout [26]. Regarding the relationship between UA and longterm prognosis, the data also supported the relationship between elevated UA and long-term prognosis in patient with acute coronary syndromes and treated with PCI [27]. Therefore, whether hyperuricemia is still a predictor of poor long-term prognosis after adjusting for CI-AKI should be studied in STEMI patients receiving pPCI.

Although the pathophysiological mechanisms of adverse reactions to hyperuricemia have not been fully elucidated, it appears to be multifactorial. In experimental models, hyperuricemia was linked to a variety of proatherogenic processes, including increased oxidative stress [28], vascular smooth muscle cell proliferation [29], inflammation [30], and endothelial dysfunction [31].

\section{Limitations}

Our current secondary analysis is subject to the following restrictions. First, its sensitivity was lower due to the definition of $>0.5 \mathrm{mg} / \mathrm{dL}$ because it was less selective for patients with higher risk of mortality and morbidity. Second, single baseline SUA measurements were used to predict CI-AKI and long-term mortality. However, a 
number of previous studies have used this method as well. Third, the measurement of serum creatinine (SCr) was standardized at $72 \mathrm{~h}$ after pPCI rather than at random, which might lead to the ignorance at the increase in delayed SCr $(>72 \mathrm{~h})$. Finally, it is a secondary analysis that is not capable of testifying a causal relationship.

In conclusion, our study will determine the association between hyperuricemia and contrast-induced acute kidney injury (CI-AKI) after primary percutaneous coronary intervention ( $\mathrm{pPCI}$ ) and will identify if hyperuricemia is a risk factor for long-term death after adjusting for CIAKI.

\section{Trial status}

The first patient was included in the RESCIND -1 ATTEMPT Trial (Protocol version 2.0, 11 June 2014) on 1 July 2014 and expected to complete recruitment in December 2018. As of October 24, 2018, recruitment is ongoing with 555 patients randomized at 15 centers in China.

\section{Abbreviations}

Cl-AKl: Contrast-induced acute kidney injury; pPCl: Primary percutaneous coronary intervention; STEMI: ST-segment elevation myocardial infarction; SUA: Serum uric acid; SCr: Serum creatinine

\section{Acknowledgements}

The authors are solely responsible for the design and conduct of this study, all study analyses, and the drafting and editing of the paper and its final contents. Special thanks go to the project managers Mr. Yongquan Yang and Ms. Lingfen Chen for their considerable time and effort in the ATTEMPT study.

\section{Authors' contributions}

NT and YL are the principal investigators. WG, FES, and SQC are the main coordinator of the project. GLS and $J$ participated in the development of the protocol. LZ and JYC provided the basic suggestions for the study. WG drafted the manuscript of the present paper. All the authors were involved in the manuscript's revision and approved the final version.

\section{Funding}

Guangdong society of interventional cardiology (grant no. GSIC20140526); Science and Technology Planning Project of Guangdong Province (grant No.2014B070706010); The National Science Foundation for Young Scientist of China (grant no. 81500520).

\section{Availability of data and materials}

Data sharing not applicable to this article as no datasets were generated or analyzed during the current study.

\section{Ethics approval and consent to participate}

The study protocol has been approved by the Guangdong Provincial People's Hospital Ethics Committee. All the participants will sign an informed consent form. Summary data will be used only for statistical analysis to ensure that personal information is not leaked.

\section{Consent for publication}

Not applicable.

\section{Competing interests}

The authors declare that they have no competing interests.

\section{Author details}

'Department of Cardiology, Guangdong Provincial Key Laboratory of Coronary Heart Disease Prevention, Guangdong Cardiovascular Institute,
Guangdong Provincial People's Hospital, Guangdong Academy of Medical Sciences, Guangzhou 510080, China. ${ }^{2}$ Guangdong Provincial Geriatrics Institute, Guangdong Provincial People's Hospital, Guangdong Academy of Medical Sciences, Guangzhou 510100, China.

Received: 14 April 2019 Accepted: 12 June 2020

Published online: 24 June 2020

\section{References}

1. Marenzi G, Lauri G, Assanelli E, et al. Contrast-induced nephropathy in patients undergoing primary angioplasty for acute myocardial infarction. J Am Coll Cardiol. 2004;44:1780-5.

2. Goldberg A, Hammerman H, Petcherski S, et al. Inhospital and 1-year mortality of patients who develop worsening renal function following acute ST-elevation myocardial infarction. Am Heart J. 2005;150:330-7.

3. Mehran R, Aymong ED, Nikolsky E, et al. A simple risk score for prediction of contrast-induced nephropathy after percutaneous coronary intervention: development and initial validation. J Am Coll Cardiol. 2004;44:1393-9.

4. Kanbay M, Segal M, Afsar B, et al. The role of uric acid in the pathogenesis of human cardiovascular disease. Heart. 2013;99:759-66.

5. Borghi C, Rosei EA, Bardin T, et al. Serum uric acid and the risk of cardiovascular and renal disease. J Hypertens. 2015;33:1729-41 discussion 41.

6. Guo W, Liu Y, Chen J-Y, et al. Hyperuricemia is an independent predictor of contrast-induced acute kidney injury and mortality in patients undergoing percutaneous coronary intervention. Angiology. 2015;66:721-6.

7. Liu Y, Tan N, Chen J, et al. The relationship between hyperuricemia and the risk of contrast-induced acute kidney injury after percutaneous coronary intervention in patients with relatively normal serum creatinine. Clinics (Sao Paulo). 2013:68:19-25.

8. Barbieri L, Verdoia M, Schaffer A, et al. Uric acid levels and the risk of contrast induced nephropathy in patients undergoing coronary angiography or PCI. Nutr Metab Cardiovasc Dis. 2015;25:181-6.

9. Kanbay M, Solak Y, Afsar B, et al. Serum uric acid and risk for acute kidney injury following contrast. Angiology. 2017;68:132-44.

10. Park S-H, Shin W-Y, Lee E-Y, et al. The impact of hyperuricemia on inhospital mortality and incidence of acute kidney injury in patients undergoing percutaneous coronary intervention. Circ J. 2011;75:692-7.

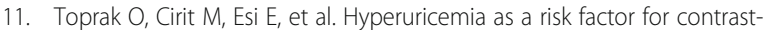
induced nephropathy in patients with chronic kidney disease. Catheter Cardiovasc Interv. 2006;67:227-35.

12. Toprak O, Cirit M, Yesil M, et al. Impact of diabetic and pre-diabetic state on development of contrast-induced nephropathy in patients with chronic kidney disease. Nephrol Dial Transplant. 2007;22:819-26.

13. Zuo T, Jiang L, Mao S, et al. Hyperuricemia and contrast-induced acute kidney injury: a systematic review and meta-analysis. Int J Cardiol. 2016;224: 286-94.

14. Karabulut A, Sahin I, Ilker Avci I, et al. Impact of serum alkaline phosphatase level on the pathophysiologic mechanism of contrast-induced nephropathy. Kardiol Pol. 2014;72:977-82.

15. Kowalczyk J, Francuz P, Swoboda R, et al. Prognostic significance of hyperuricemia in patients with different types of renal dysfunction and acute myocardial infarction treated with percutaneous coronary intervention. Nephron Clin Pract. 2010;116:c114-22.

16. Elbasan Z, Sahin DY, Gur M, et al. Contrast-induced nephropathy in patients with ST elevation myocardial infarction treated with primary percutaneous coronary intervention. Angiology. 2014;65:37-42.

17. Mendi MA, Afsar B, Oksuz F, et al. Uric acid is a useful tool to predict contrast-induced nephropathy. Angiology. 2016;68:627-32.

18. Saritemur M, Turkeli M, Kalkan K, et al. Relation of uric acid and contrastinduced nephropathy in patients undergoing primary percutaneous coronary intervention in the ED. Am J Emerg Med. 2014;32:119-23.

19. Liu Y, Chen JY, Huo Y, et al. Aggressive hydraTion in patients with STElevation Myocardial infarction undergoing Primary percutaneous coronary intervention to prevenT contrast-induced nephropathy (ATTEMPT): Study design and protocol for the randomized, controlled trial, the ATTEMPT, RESCIND 1 (First study for REduction of contraSt-induCed nephropathy followINg carDiac catheterization) trial. Am Heart J. 2016;172:88-95.

20. Tan N, Liu Y, Chen JY, et al. Use of the contrast volume or grams of iodineto-creatinine clearance ratio to predict mortality after percutaneous coronary intervention. Am Heart J. 2013;165:600-8. 
21. Narula A, Mehran R, Weisz G, et al. Contrast-induced acute kidney injury after primary percutaneous coronary intervention: results from the HORIZONS-AMl substudy. Eur Heart J. 2014;35:1533-40.

22. Zoungas S, Ninomiya T, Huxley R, et al. Systematic review: sodium bicarbonate treatment regimens for the prevention of contrast-induced nephropathy. Ann Intern Med. 2009;151:631-8.

23. Bhatt H, Turkistani A, Sanghani D, Julliard K, Fernaine G. Do cardiovascular risk factors and coronary SYNTAX score predict contrast volume use during cardiac catheterization? Angiology. 2015;66:933-40

24. Krishnan E, Svendsen $\mathrm{K}$, Neaton JD, et al. Long-term cardiovascular mortality among middle-aged men with gout. Arch Intern Med. 2008;168:1104-10.

25. Kuo CF, See LC, Luo SF, et al. Gout: an independent risk factor for all-cause and cardiovascular mortality. Rheumatology (Oxford). 2010;49:141-6.

26. Pagidipati NJ, Hess CN, Clare RM, et al. An examination of the relationship between serum uric acid level, a clinical history of gout, and cardiovascular outcomes among patients with acute coronary syndrome. Am Heart J. 2017;187:53-61.

27. Tscharre M, Herman R, Rohla M, et al. Uric acid is associated with long-term adverse cardiovascular outcomes in patients with acute coronary syndrome undergoing percutaneous coronary intervention. Atherosclerosis. 2018;270: 173-9.

28. Sautin YY, Nakagawa T, Zharikov S, et al. Adverse effects of the classic antioxidant uric acid in adipocytes: NADPH oxidase-mediated oxidative/ nitrosative stress. Am J Physiol Cell Physiol. 2007;293:C584-96.

29. Corry DB, Eslami P, Yamamoto K, et al. Uric acid stimulates vascular smooth muscle cell proliferation and oxidative stress via the vascular reninangiotensin system. J Hypertens. 2008;26:269-75.

30. Kang DH, Park SK, Lee IK, et al. Uric acid-induced C-reactive protein expression: implication on cell proliferation and nitric oxide production of human vascular cells. J Am Soc Nephrol. 2005;16:3553-62.

31. Kanbay M, Yilmaz MI, Sonmez A, et al. Serum uric acid level and endothelial dysfunction in patients with nondiabetic chronic kidney disease. Am J Nephrol. 2011;33:298-304.

\section{Publisher's Note}

Springer Nature remains neutral with regard to jurisdictional claims in published maps and institutional affiliations.

Ready to submit your research? Choose BMC and benefit from:

- fast, convenient online submission

- thorough peer review by experienced researchers in your field

- rapid publication on acceptance

- support for research data, including large and complex data types

- gold Open Access which fosters wider collaboration and increased citations

- maximum visibility for your research: over $100 \mathrm{M}$ website views per year

At $\mathrm{BMC}$, research is always in progress.

Learn more biomedcentral.com/submissions 